\title{
Connecting Faith and Health: Improving Health Outcomes Through Congregant Networks
}

\author{
Meredith Campbell ${ }^{1}$, Jacob Christenson ${ }^{2,3}$, David Craig ${ }^{4}$ \\ ${ }^{1}$ Indiana University School of Medicine; ${ }^{2}$ Indiana Minority Health Coalition; ${ }^{3}$ Indiana Clinical and \\ Translational Sciences Institute; ${ }^{4}$ Indiana University- Purdue University Indianapolis, \\ Department of Religious Studies
}

Background and Hypothesis: People living in areas of high social vulnerability face health disparities in part due to disconnection. Health institutions recognize the importance of treating whole persons but are disconnected from local knowledge of community health assets and social and cultural barriers. People in health-challenged neighborhoods may experience disconnection and distrust in seeking the many resources and services needed for good health. Congregations are longstanding anchor institutions in marginalized communities. They build trusted relationships among members through shared values and care for wellness. Sometimes they extend these connections through on-the-ground service to neighboring communities. We hypothesize that a network of congregations can build trusted connections and share local knowledge and cultural competence to improve health outcomes and holistic wellness in vulnerable neighborhoods.

Methods: Community-engaged participatory research requires reciprocity in mapping assets, identifying priorities, narrating shared values, designing projects and messaging results. We developed mixed-methods tools to ensure community expertise drives an iterative research process. Methods include: an initial health and wellness survey, follow-up wellness interviews to collect stories and assets, an online learning community for 14 congregations with monthly focus groups, and visual maps of health data and wellness assets.

Results: While the study is ongoing, preliminary survey data demonstrates that congregations have local knowledge of issues affecting health and wellness among their congregants and the surrounding neighborhood. Their preexisting support for health and wellness through community outreach programs indicates building on their current network could expand their reach and improve health and wellness in vulnerable neighborhoods.

Conclusion and Potential Impact: Successful community engaged research prioritizes iterative methods that allow community participants to use their voice and tell their stories. Congregations' practice of shared values and knowledge of lived experience can forge connections for greater resilience and health supports in socially vulnerable communities. 